\title{
LIEUX DE REPOS DIURNES DE GLOSSINA PALPALIS PALPALIS (ROBINEAU-DESVOIDY) DANS UNE ZONE PREFORESTIERE DE COTE D'IVOIRE*
}

\author{
A. SEKETELI ${ }^{+}$et F. A. S. KUZOE ${ }^{\neq}$ \\ Project OMS de Recherche et Formation en matière de \\ Trypanosomiases humaines, Daloa, Côte d'Ivoire
}

(Received 17 September 1991; accepted 1 February 1992)

\begin{abstract}
Résumé-Des études sur les lieux de repos diurnes de Glossina palpalis palpalis ont été menées d'octobre 1981 à décembre 1982 dans le foyer de trypanosomiase humaine de Bouaflé (Côte d'Ivoire). 1382 glossines (970 mâles et 412 femelles), ont été observées au repos dans leurs habitats naturels après 757 heures de recherche. Quelle que soit la saison, plus de $80 \%$ des mouches étaient trouvées au repos sur des lianes (Acacia pennata en particulier), sur des caféiers (Coffea indica), sur des composées représentées par l'espèce Eupatorium odoratum.

La quasi totalité des mouches observées se reposaient de préférence sur la face inférieure des parties ligneuses des plantes choisies, à des hauteurs comprises entre $10 \mathrm{~cm}$ et $2,5 \mathrm{~m}$ du sol. Les diamètres des supports ligneuses allaient de $0,1 \mathrm{~cm}$ à $8 \mathrm{~cm}$. Il convient de noter que quelle que soit la saison, plus de $50 \%$ des mouches se reposaient à une hauteur inférieure à $50 \mathrm{~cm}$ et que plus de $90 \%$ d'entre elles étaient observées sur des supports de diamètres inférieures ou égaux à $3 \mathrm{~cm}$.

Sur la végétation bordant les villages ou les différentes voies d'accès, les glossines ont toujours été trouvées au repos en profonfeur dans cette végétation à des distances comprises entre $\mathbf{1}$ et $5 \mathbf{m}$ des lisières.

Environ $27 \%$ des mouches capturées au repos ont été trouvées gorgées ou en phase intermédiare. La capture des glossines au repos dans leurs habitats constitue donc dans la zone d'étude, un moyen privilégié pour la collecte de repas de sang frais en vue de l'identification des hôtes naturels de $G$. palpalis palpalis.

Les implications practiques d'une telle étude sont discutées et des recommandations sont formulées pour l'application sélective d'insecticides rémanents contre les vecteurs de la trypanosomiase humaine dans le foyer de Bouaflé.
\end{abstract}

\section{Mots Clés: Glossines, Glossina palpalis palpalis, lieux de repos}

\begin{abstract}
The diurnal resting sites of Glossina palpalis palpalis were studied from October 1981 to December 1982 in the human trypanosomiasis focus at Bouafle (Ivory Coast). A total of 1382 resting tsetse (970 males and 412 females), were found after $757 \mathrm{hr}$ of search in their natural habitats.

At any season, over $80 \%$ of the flies were resting on lianas (Acacia pennata in particular), on coffee trees (Coffea indica) and on Eupatorium odoratum.

Almost all the flies showed a preference for resting on the underside of woody parts of plants selected. The heights of the resting sites ranged from $10 \mathrm{~cm}$ to $2.5 \mathrm{~m}$ above the ground and their diameters ranged from $0.1 \mathrm{~cm}$ to $8 \mathrm{~cm}$. It must be stressed that both in the dry and in the rainy seasons, more than $50 \%$ of the flies rested below $50 \mathrm{~cm}$ from the ground and over $90 \%$ of these favoured stems and branches measuring less than $3 \mathrm{~cm}$ in dia.
\end{abstract}

\footnotetext{
${ }^{*}$ Cette étude a été financée par le Programme Spécial PNUD/Banque Mondiale/OMS de Recherche et de Formation concernant les maladies tropicales.

${ }^{+}$Adresse pour correspondance : Dr. A. Sékétéli, OMS/OCP, B.P. 549, Ouagadougou, Burkina Faso.

${ }^{\ddagger}$ Adresse actuelle : Organisation Mondiale de la Sante, Avenue Appia, Geneve, Suisse.
} 
In the vegetation surrounding villages or along the main access roads and bush pathways, $G$. p. palpalis were found resting at $1 \mathrm{~m}$ to $5 \mathrm{~m}$ from the edge of its vegetation habitat.

About $27 \%$ of flies were engorged or showed at least red blood meals in their guts. The catching of resting flies therefore, appears to be an excellent way of collecting fresh blood meals for identification of the natural vertebrate hosts of $G$. p. palpalis in the study area.

The practical interest of the study is discussed and recommendations made for the selective application of residual insecticides in the tsetse habitats to control the vectors of sleeping sickness in the Bouafle focus.

Key Words: Glossina, Glossina palpalis palpalis, resting sites

\section{INTRODUCTION}

L'un des objectifs des études entomoépidémiologiques conduites de 1980 à 1985 dans le foyer de trypanosomiase humaine de Bouaflé, Côte d'Ivoire, dans le cadre du Projet OMS de Recherche Appliquées sur la Trypanosomiase(Projet TDR 308), était la mise au point de méthodes de lutte antiglossines simples, efficaces, peu onéreux et le moins toxiques possible pour la faune non-cible.

C'est ainsi que furent ménés contre les vecteurs de la maladie du sommeil dans ledit foyer, des essais d'épandage au sol de nouvelles formulations ou de formulations déjà existantes de pyréthrinoïdes de synthèse en utilisant des atomiseurs portatifs couramment utilisés par les villageois de la région à des fins agricoles (Sékétéli et Kuzoe, 1983, 1986 ; Sékétéli et al., 1985).

Or, l'utilisation rationnelle de ces insecticides par leur appplication sélective et discriminative était subordonnée à une bonne connaissance des lieux de repos des glossines dans leurs habitats naturels. C'est pourquoi nous avions entrepris d'octobre 1981 à décembre 1982, la recherche des lieux de repos diurnes de Glossina palpalis palpalis, principal vecteur de la maladie du sommeil dans la zone d'étude.

Le présent papier fait état des résultats de ces études.

\section{MATERIEL ET METHODES}

Comme déjà mentionné en introduction, lesétudes se sont déroulées d'octobre 1981 à décembre 1982 en Côte d'Ivoire dans la région de Bouaflé dont la présentation a déjà été faite dans un précédent article (Sékétéli et Kuzoe, 1983).

Rappelons simplement que cette zone de Bouaflé est située dans le domaine guinéen, en zone de forêt semi-décidue du secteur mésophile (Guillaumet et Adjonohoun, 1971) couramment appelé secteur préforestier. Elle est caractérisée par une végétation de type mosaïque, avec des zones de forêts primaires presque toutes dégradées par l'homme, entrecoupées de plantations et de savane arborée.

La recherche des glossines au repos s'est opérée dans les localités de Kouassi-Périta, Dégbézéré, Koudougou et plus précisement dans la végétation

Tableau 1. Répartition en fonction des saisons, du nombre de G. palpalis palpalis observées et capturées de jour au repos dans la région de Bouaflé, Côte d'Ivoire

\begin{tabular}{|c|c|c|c|c|c|c|c|c|}
\hline \multirow[b]{2}{*}{ Saison } & \multirow{2}{*}{$\begin{array}{l}\mathrm{Nb} \text {. Heures } \\
\text { de recherches }\end{array}$} & \multicolumn{3}{|c|}{$\begin{array}{c}\text { Nb. mouches } \\
\text { observées }\end{array}$} & \multirow{2}{*}{$\begin{array}{c}\text { Sex-ratio }{ }^{*} \\
\text { (=\% femelles) }\end{array}$} & \multicolumn{3}{|c|}{$\begin{array}{l}\text { Nb. mouches } \\
\text { capturées }\end{array}$} \\
\hline & & M & $F$ & $\mathrm{M}+\mathrm{F}$ & & $\mathbf{M}$ & $\mathrm{F}$ & $\mathrm{M}+\mathrm{F}$ \\
\hline $\begin{array}{l}\text { Saison sèche froide } \\
\text { (nov. dec. janv.) }\end{array}$ & 190 & 216 & 102 & 318 & 32,08 & $\begin{array}{c}128 \\
(59,26)\end{array}$ & $\begin{array}{c}63 \\
(61,76) \\
\end{array}$ & $\begin{array}{c}191 \\
(60,06) \\
\end{array}$ \\
\hline $\begin{array}{l}\text { Saison sèche chaude } \\
\text { (fév. marc. avr.) }\end{array}$ & 181 & 186 & 107 & 293 & 36,52 & $\begin{array}{c}141 \\
(75,81)\end{array}$ & $\begin{array}{c}65 \\
(60,75) \\
\end{array}$ & $\begin{array}{c}206 \\
(70,31) \\
\end{array}$ \\
\hline $\begin{array}{l}\text { Saison pluvieuse } \\
\text { (mai à octobre) }\end{array}$ & 386 & 568 & 203 & 771 & 26,33 & $\begin{array}{c}392 \\
(69,01)\end{array}$ & $\begin{array}{c}148 \\
(72,91)\end{array}$ & $\begin{array}{c}540 \\
(70,04)\end{array}$ \\
\hline Total & 757 & 970 & 412 & 1382 & 29,81 & $\begin{array}{c}662 \\
(68,18)\end{array}$ & $\begin{array}{c}276 \\
(66,99)\end{array}$ & $\begin{array}{c}938 \\
(67,82)\end{array}$ \\
\hline
\end{tabular}

$\mathrm{M}=$ mâle. $\mathrm{F}=$ femelle. $\mathrm{M}+\mathrm{F}=$ mâle et femelle.

Les chiffres entre parenthèses indiquent les pourcentages de glossines capturées par rapport au nombre de glossines observées dans chaque catégorie $\mathrm{M}, \mathrm{F}$ ou $\mathrm{M}+\mathrm{F}$.

*Le sex-ratio est défini comme le pourcentage de femelles, ce pourcentage étant calculé par rapport au nombre total (M+F) de glossines observées au cours de la saison considérée. 
bordant ces villages, dans les champs de cultures vivrières, les plantations de café et de cacao, dans la végétation longeant les pistes empruntées par les villageois lors de leurs activités domestiques ou champêtres.

Pour la localisation géographique des villages cités voir Sékétéli et al. (1985).

Les glossines au repos dans leurs habitats naturels étaient recherchées à vue et capturées à l'aide de tubes de verre.

Avant la capture, la mouche était attentivement observée et son nom d'espèce ainsi que son sexe notés. On ne prenait en compte que les mouches réellement au repos c'est-à-dire celles qui étaient sur le support avant l'arrivée de l'observateur et qui, si elles n'étaient pas perturbées, pouvaient se laisser observer pendant au moins cinq minutes. Onéliminait ainsi la fraction de mouches qui au cours de leur vol marquaient sur n'importe quel support, un bref temps d'arrêt.

Les noms communs des plantes hébergeant les glossines ainsi que les parties de ces plantes (tronc, branche, feuille) qui servaient de support de repos étaient enregistrés. D'autres informations tels que le diamètre des organes végétaux, leur hauteur à partir du sol, l'état des feuilles (mortes ou vertes), la position de la mouche au repos, ont été également notées.

Les recherches se sont déroulées aux heures les plus chaudes de la journée, de 11 à $15 \mathrm{~h} 30$.

L'équipe de recherche comprenait 4 à 6 agents qui évoluaient séparément dans les biotopes mentionnés, munis de tubes de capture, de cordes, de règles graduées, de carnets de notes.

Les mouches capturées ont été disséquées le même jour au laboratoire pour la collecte de repas de sang frais en vue de l'identification ultérieure des hôtes naturels du vecteur.

\section{RESULTATS}

Répartition par saison du nombre de glossines observées et capturées de jour au repos

757 heures de recherche ( 371 heures en saison sèche et 386 en saison pluvieuse) ont été enregistrées.

Comme l'indique le Tableau 1, un total de 1.382 glossines (611 en saison sèche et 771 en saison pluvieuse) furent observées au repos.

Surces 1.382 mouches observées, 938 soit environ $68 \%$, ont pu être capturés à l'aide des tubes.

Notons que dans l'ensemble la proportion de glossines capturées par rapport aux glossines observées est à peu près la même chez les deux sexes: environ $68 \%$ des mâles observés au repos étaient capturés contre $67 \%$ de capturées chez les femelles.

Environ $30 \%$ seulement des 1.382 glossines observées au repos étaient des femelles, ce pourcentage étant plus faible en saison des pluies (26\%) qu'en saison sèche où il était de $34 \%$ environ (voir Tableau 1).

Identité des plantes hôtes et comportement de $\mathrm{G}$. palpalis palpalis au repos

Les principales plantes ayant hébergéles glossines sont : le caféier (Coffea indica) (Fig. 1.); le cacaoyer (Theobroma cacao); le palmier à huile (Eleais guineensis), l'ananas (Ananas comosus); le gombo (Hibiscus esculentus) ; des graminées avec l'espèce Pennisetum purpureum (Fig. 2); des lianes appartenant en grande partie à la famille des mimosacées avec comme espèce dominante: Acacia pennata (Fig. 3); des cesalpiniacées avec les espèces Caesalpinia pulcherima et Caesalpinia bonduc; des Euphorbiacées avec l'espece Ricinus communis; des Composées avec l'espèce Eupatorium odoratum communément appelées "Sekou-Toure" dans la zone d'étude (Fig. 4); des Bombacacées avec l'espèce Ceiba pentandra; des fougères.

Pour leur repos, les mouches ont choisi presque exclusivement les organes ligneux vivants ou morts (branches, brindilles) des plantes citées. Une seule mouche a été capturée au repos sur une feuille de gombo. Par ailleurs, les branches et brindilles inclinées par rapport au plan du sol sont choisies par les glossines de préférence à celles qui sont verticles (Figs. 1-4).

La quasi totalité des mouches observées se reposaient à la face inférieure de ces parties ligneuses inclinées, en position parallèle ou légèrement oblique par rapport au support.

Les endroits choisis par les glossines pour leur repos ont toujours été à l'abri du soleil si bien que nous n'avons jamais observé de mouches au repos sur les bordures ensoleillées de la végétation bordant les villages ou les différentes voies d'accès. Elles ont toujours été capturées en profondeur dans la végétation à des distances comprises entre $1 \mathrm{~m}$ et $5 \mathrm{~m}$ des lisières.

Aucune mouche n'a été non plus trouvée au repos sur les arbres isolés situés a l'intérieur des villages et procurant de l'ombre aux habitants.

Comme pour se protéger de ses ennemis naturels, les parties ligneuses des plantes choisies par la mouche pour son repos ont à peuprès la même couleur que celle de la glossine elle-même ce qui rend encore plus difficile la détection à vue de cette dernière dans la végétation.

Répartition des mouches au repos en fonction de la plante hôte: variations saisonières

Comme l'indique le Tableau 2, la gamme de plantes qui, en saison pluvieuse s'offrent à la glossine 

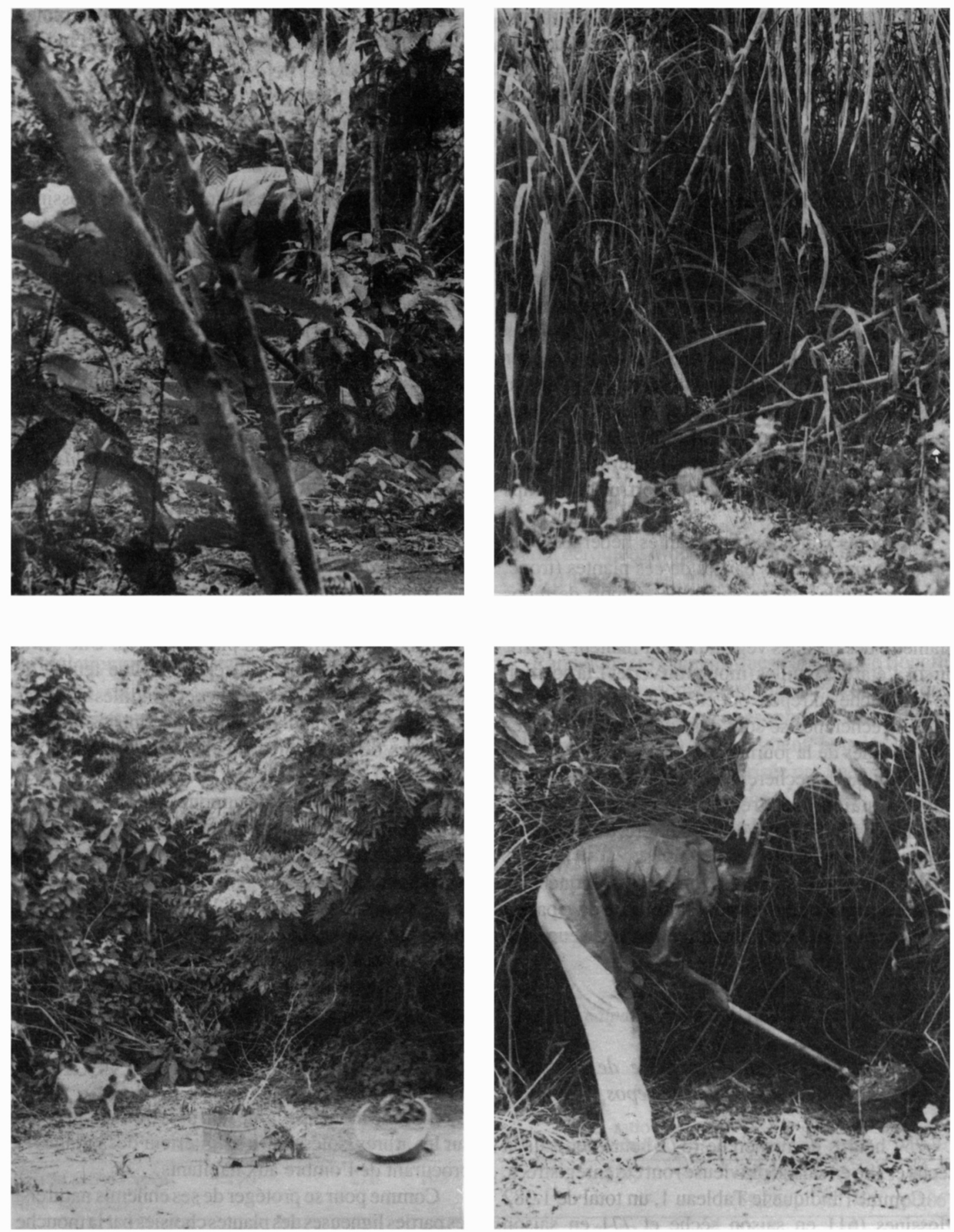

Figs 1-4. Lieux de repos diurnes de G. p. palpalis constitués de branches inclinées de caféiers (1), de tiges inclinées de Pennisetum purpureum (2). De lianes (Acacia pennata) servant aussi d'abris aux porcs domestiques, hôtes préférentiels du vecteur (3) et de tiges inclinées d'Eupatorium odoratum sous lesquelles les glossines femelles vont également déposer leurs larves (4).

Les flèches indiquent les parties des plantes choisies par les mouches pour leur repos. 
Tableau 2. Répartition en fonction de la plante hôte et par saison, du nombre de G. palpalis palpalis observées de jour au repos dans la région de Bouaflé, Côte d'Ivoire

\begin{tabular}{|c|c|c|c|c|c|c|c|c|c|}
\hline \multirow[b]{2}{*}{ Plante hôte } & \multicolumn{3}{|c|}{$\begin{array}{l}\text { Saison sèche froide } \\
\text { (nov. déc. janv.) }\end{array}$} & \multicolumn{3}{|c|}{$\begin{array}{c}\text { Saison sèche chaude } \\
\text { (fév. mars. avril) }\end{array}$} & \multicolumn{3}{|c|}{$\begin{array}{l}\text { Saison pluvieuse } \\
\text { (mai à octobre) }\end{array}$} \\
\hline & $\begin{array}{c}M \\
n=216 \\
\end{array}$ & $\begin{array}{c}\mathrm{F} \\
n=102\end{array}$ & $\begin{array}{c}\mathrm{M}+\mathrm{F} \\
n=318 \\
\end{array}$ & $\begin{array}{c}\mathrm{M} \\
n=186 \\
\end{array}$ & $\begin{array}{c}\mathrm{F} \\
n=107\end{array}$ & $\begin{array}{c}\mathrm{M}+\mathrm{F} \\
n=293\end{array}$ & $\begin{array}{c}M \\
n=568\end{array}$ & $\begin{array}{c}\mathrm{F} \\
n=203\end{array}$ & $\begin{array}{c}\mathrm{M}+\mathrm{F} \\
n=771\end{array}$ \\
\hline Lianes & $\begin{array}{c}132 \\
(61,11)\end{array}$ & $\begin{array}{c}54 \\
(52,94)\end{array}$ & $\begin{array}{c}186 \\
(58,49)\end{array}$ & $\begin{array}{c}179 \\
(96,24)\end{array}$ & $\begin{array}{c}104 \\
(97,20)\end{array}$ & $\begin{array}{c}283 \\
(96,59)\end{array}$ & $\begin{array}{c}199 \\
(35,04)\end{array}$ & $\begin{array}{c}97 \\
(47,78)\end{array}$ & $\begin{array}{c}296 \\
(38,39)\end{array}$ \\
\hline Coffea indica & $\begin{array}{c}45 \\
(20,83)\end{array}$ & $\begin{array}{c}28 \\
(27,45)\end{array}$ & $\begin{array}{c}73 \\
(22,96)\end{array}$ & $\begin{array}{c}0 \\
(0)\end{array}$ & $\begin{array}{c}1 \\
(0,93)\end{array}$ & $\begin{array}{c}1 \\
(0,34)\end{array}$ & $\begin{array}{c}263 \\
(46,30)\end{array}$ & $\begin{array}{c}60 \\
(29,56)\end{array}$ & $\begin{array}{c}323 \\
(41,89)\end{array}$ \\
\hline $\begin{array}{l}\text { Eupatorium } \\
\text { odoratum }\end{array}$ & $\begin{array}{c}13 \\
(6,02)\end{array}$ & $\begin{array}{c}5 \\
(4,90)\end{array}$ & $\begin{array}{c}18 \\
(5,66)\end{array}$ & $\begin{array}{c}5 \\
(2,68)\end{array}$ & $\begin{array}{c}2 \\
(1,87)\end{array}$ & $\begin{array}{c}7 \\
(2,39)\end{array}$ & $\begin{array}{c}19 \\
(3,34)\end{array}$ & $\begin{array}{c}8 \\
(3,94)\end{array}$ & $\begin{array}{c}27 \\
(3,50)\end{array}$ \\
\hline $\begin{array}{l}\text { Eleais } \\
\text { guineensis }\end{array}$ & $\begin{array}{c}6 \\
(2,78)\end{array}$ & $\begin{array}{c}5 \\
(4,90)\end{array}$ & $\begin{array}{c}11 \\
(3,46)\end{array}$ & $\begin{array}{c}1 \\
(0,54)\end{array}$ & $\begin{array}{c}0 \\
(0)\end{array}$ & $\begin{array}{c}1 \\
(0,34)\end{array}$ & $\begin{array}{c}1 \\
(0,18)\end{array}$ & $\begin{array}{c}3 \\
(1,48)\end{array}$ & $\begin{array}{c}4 \\
(0,52)\end{array}$ \\
\hline $\begin{array}{l}\text { Ananas } \\
\text { comosus }\end{array}$ & $\begin{array}{c}7 \\
(3,24)\end{array}$ & $\begin{array}{c}2 \\
(1,96)\end{array}$ & $\begin{array}{c}9 \\
(2,93)\end{array}$ & $\begin{array}{c}1 \\
(0,54)\end{array}$ & $\begin{array}{c}0 \\
(0)\end{array}$ & $\begin{array}{c}1 \\
(0,34)\end{array}$ & - & - & - \\
\hline $\begin{array}{l}\text { Caesalpinia } \\
\text { pulcherima }\end{array}$ & $\begin{array}{c}0 \\
(0)\end{array}$ & $\begin{array}{c}1 \\
(0,98)\end{array}$ & $\begin{array}{c}1 \\
(0,31)\end{array}$ & $\begin{array}{l}- \\
-\end{array}$ & - & - & - & $\begin{array}{l}- \\
-\end{array}$ & - \\
\hline $\begin{array}{l}\text { Pennisetum } \\
\text { purpureum }\end{array}$ & - & - & - & - & - & - & $\begin{array}{c}25 \\
(4,40)\end{array}$ & $\begin{array}{c}16 \\
(7,88)\end{array}$ & $\begin{array}{c}41 \\
(5,32)\end{array}$ \\
\hline $\begin{array}{l}\text { Theobroma } \\
\text { cacao }\end{array}$ & - & - & $\begin{array}{l}- \\
-\end{array}$ & $\begin{array}{l}- \\
-\end{array}$ & - & $\begin{array}{l}- \\
-\end{array}$ & $\begin{array}{c}11 \\
(1,93)\end{array}$ & $\begin{array}{c}5 \\
(2,46)\end{array}$ & $\begin{array}{c}16 \\
(2,08)\end{array}$ \\
\hline $\begin{array}{l}\text { Caesalpina } \\
\text { bonduc }\end{array}$ & - & $\begin{array}{l}- \\
-\end{array}$ & - & - & $\begin{array}{l}- \\
-\end{array}$ & $\begin{array}{l}- \\
-\end{array}$ & $\begin{array}{c}1 \\
(0,18)\end{array}$ & $\begin{array}{c}1 \\
(0,49)\end{array}$ & $\begin{array}{c}2 \\
(0,26)\end{array}$ \\
\hline $\begin{array}{l}\text { Ricinus } \\
\text { communis }\end{array}$ & - & $\begin{array}{l}- \\
-\end{array}$ & - & - & - & $\begin{array}{l}- \\
-\end{array}$ & $\begin{array}{c}0 \\
(0)\end{array}$ & $\begin{array}{c}2 \\
(0,99)\end{array}$ & $\begin{array}{c}2 \\
(0,26)\end{array}$ \\
\hline $\begin{array}{l}\text { Hibiscus } \\
\text { esculentus }\end{array}$ & - & - & - & - & $\begin{array}{l}- \\
-\end{array}$ & - & $\begin{array}{c}0 \\
(0)\end{array}$ & $\begin{array}{c}1 \\
(0,49)\end{array}$ & $\begin{array}{c}1 \\
(0,13)\end{array}$ \\
\hline $\begin{array}{l}\text { Ceiba } \\
\text { pentendra }\end{array}$ & - & - & - & - & - & $\begin{array}{l}- \\
-\end{array}$ & $\begin{array}{c}1 \\
(0,18)\end{array}$ & $\begin{array}{c}0 \\
(0)\end{array}$ & $\begin{array}{c}1 \\
(0,13)\end{array}$ \\
\hline Fougères & - & $\begin{array}{l}- \\
-\end{array}$ & $\begin{array}{l}- \\
-\end{array}$ & $\begin{array}{l}- \\
-\end{array}$ & $\begin{array}{l}- \\
-\end{array}$ & - & $\begin{array}{c}3 \\
(0,53)\end{array}$ & $\begin{array}{c}2 \\
(0,99)\end{array}$ & $\begin{array}{c}5 \\
(0,65)\end{array}$ \\
\hline Indéterminées & $\begin{array}{c}13 \\
(6,02)\end{array}$ & $\begin{array}{c}7 \\
(6,87)\end{array}$ & $\begin{array}{c}20 \\
(6,29)\end{array}$ & - & - & - & $\begin{array}{c}45 \\
(7,92)\end{array}$ & $\begin{array}{c}8 \\
(3,94)\end{array}$ & $\begin{array}{c}53 \\
(6,87)\end{array}$ \\
\hline
\end{tabular}

$\mathrm{M}=$ mâle; $\mathrm{F}=$ femelle; $\mathrm{M}+\mathrm{F}=$ mâle et femelle.

$n=$ nombre total de glossines mâles, femelles ou mâles et femelles, observées durant la saison considérée.

Les chiffres entre parenthèses représentent les pourcentages de glossines observées au repos, ces pourcentages étant, dans chaque catégorie (M, F, M+F), calculés par rapport aux valeurs correspondantes de $n$.

pour son repos est plus vaste et variée que celle dont elle bénéficie en saisons sèches froide et chaude. Malgré cela, les mouches, quelle que soit la saison, ont été observées plus souvent au repos sur les lianes (Acacia pennata en particulier), les caféiers (Coffea indica) et sur Eupatorium odoratum: c'est ainsi que ces trois groupes de plantes ont pu héberger environ 87,99 et $84 \%$ des mouches observées au repos respectivement en saison sèche froide, saison sèche chaude et saison pluvieuse.
Notons q'en saison sèche chaude (février, mars, avril), presque $99 \%$ des mouches se reposaient sur les lianes et Eupatorium odoratum.

Répartition en hauteur des mouches au repos: variations saisonnières

Dans l'ensemble, les hauteurs auxquelles étaient observées les mouches au repos se situaient entre 10 $\mathrm{cm}$ et $2,50 \mathrm{~m}$ du sol. 
Tableau 3. Répartition en fonction de la hauteur de repos et par saison, due nombre de Glossina palpalis palpalis observées de jour au repos dans la région de Bouaflé, Côte d'Ivoire

\begin{tabular}{|c|c|c|c|c|c|c|}
\hline \multirow[b]{2}{*}{ Saison } & \multirow[b]{2}{*}{ Sexe } & \multicolumn{5}{|c|}{ Nombre et proportion de glossines observées } \\
\hline & & $0-50 \mathrm{~cm}$ & $51-100 \mathrm{~cm}$ & $101-150 \mathrm{~cm}$ & $151-200 \mathrm{~cm}$ & $201-250 \mathrm{~cm}$ \\
\hline & $\begin{array}{c}\text { M } \\
n=216\end{array}$ & $\begin{array}{c}124 \\
(57,41)\end{array}$ & $\begin{array}{c}63 \\
(29,17)\end{array}$ & $\begin{array}{c}24 \\
(11,11)\end{array}$ & $\begin{array}{c}5 \\
(2,31)\end{array}$ & \\
\hline \multirow[t]{3}{*}{$\begin{array}{l}\text { Saison sèche froide } \\
\text { (nov. déc. janv.) }\end{array}$} & $\begin{array}{c}\mathrm{F} \\
n=102\end{array}$ & $\begin{array}{c}58 \\
(56,86)\end{array}$ & $\begin{array}{c}22 \\
(21,57)\end{array}$ & $\begin{array}{c}15 \\
(14,71)\end{array}$ & $\begin{array}{c}7 \\
(6,86)\end{array}$ & \\
\hline & $\begin{array}{c}M+F \\
n=318\end{array}$ & $\begin{array}{c}182 \\
(57,23)\end{array}$ & $\begin{array}{c}85 \\
(26,73)\end{array}$ & $\begin{array}{c}39 \\
(12,26)\end{array}$ & $\begin{array}{c}12 \\
(3,78)\end{array}$ & \\
\hline & $\begin{array}{c}M \\
n=186\end{array}$ & $\begin{array}{c}139 \\
(74,73)\end{array}$ & $\begin{array}{c}40 \\
(21,51)\end{array}$ & $\begin{array}{c}7 \\
(3,76)\end{array}$ & & \\
\hline \multirow[t]{3}{*}{$\begin{array}{l}\text { Saison sèche chaude } \\
\text { (fév. mars. avril) }\end{array}$} & $\begin{array}{c}\mathrm{F} \\
n=107\end{array}$ & $\begin{array}{c}76 \\
(71,03)\end{array}$ & $\begin{array}{c}27 \\
(25,23)\end{array}$ & $\begin{array}{c}4 \\
(3,74)\end{array}$ & & \\
\hline & $\begin{array}{c}M+F \\
n=293\end{array}$ & $\begin{array}{c}215 \\
(73,38)\end{array}$ & $\begin{array}{c}67 \\
(22,87)\end{array}$ & $\begin{array}{c}11 \\
(3,75)\end{array}$ & & \\
\hline & $\begin{array}{c}\mathrm{M} \\
n=568\end{array}$ & $\begin{array}{c}266 \\
(46,83)\end{array}$ & $\begin{array}{c}98 \\
(17,25)\end{array}$ & $\begin{array}{c}119 \\
(20,95)\end{array}$ & $\begin{array}{c}73 \\
(12,85)\end{array}$ & $\begin{array}{c}12 \\
(2,12)\end{array}$ \\
\hline \multirow[t]{2}{*}{$\begin{array}{l}\text { Saison pluvieuse } \\
\text { (mai à octobre) }\end{array}$} & $\begin{array}{c}F \\
n=203\end{array}$ & $\begin{array}{c}127 \\
(62,56)\end{array}$ & $\begin{array}{c}30 \\
(14,78)\end{array}$ & $\begin{array}{c}34 \\
(16,75)\end{array}$ & $\begin{array}{c}10 \\
(4,93)\end{array}$ & $\begin{array}{c}2 \\
(0,98)\end{array}$ \\
\hline & $\begin{array}{c}\mathrm{M}+\mathrm{F} \\
n=771\end{array}$ & $\begin{array}{c}393 \\
(50,97)\end{array}$ & $\begin{array}{c}128 \\
(16,60)\end{array}$ & $\begin{array}{c}153 \\
(19,84)\end{array}$ & $\begin{array}{c}83 \\
(10,77)\end{array}$ & $\begin{array}{c}14 \\
(1,82)\end{array}$ \\
\hline
\end{tabular}

$0-50 \mathrm{~cm}$ : hauteur de repos comprise entre 0 et $50 \mathrm{~cm}$ inclus; hauteur mesurée à partir du sol.

$\mathrm{M}=$ mâle $; \mathrm{F}=$ femelle; $\mathrm{M}+\mathrm{F}=$ mâle et femelle, $n=$ nombre total de glossines mâles, femelles ou mâles et femelles, observées durant la saison considérée. Les chiffres entre parenthéses représentent les pourcentages de glossines observées au repos, ces pourcentages étant, dans chaque catégorie (M, F, M+F), calculés par rapport aux valeurs correspondantes de $n$.

Tableau 4. Répartition en fonction du diametre du support ligneux de repos et par saison, du nombre de Glossina palpalis palpalis observées de jour au repos dans la région de Bouaflé, Côte d'Ivoire

\begin{tabular}{|c|c|c|c|c|c|}
\hline \multirow[b]{2}{*}{ Saison } & \multirow[b]{2}{*}{ Sexe } & \multicolumn{4}{|c|}{ Nombre et proportion de glossines observées } \\
\hline & & $0,1-1 \mathrm{~cm}$ & $1,1-3 \mathrm{~cm}$ & $3,1-5 \mathrm{~cm}$ & $5,1-8 \mathrm{~cm}$ \\
\hline & $\begin{array}{c}M \\
n=216\end{array}$ & $\begin{array}{c}32 \\
(14,81)\end{array}$ & $\begin{array}{c}178 \\
(82,41)\end{array}$ & $\begin{array}{c}5 \\
(2,32)\end{array}$ & $\begin{array}{c}1 \\
(0,46)\end{array}$ \\
\hline \multirow[t]{3}{*}{$\begin{array}{l}\text { Saison sèche froide } \\
\text { (nov. déc. janv.) }\end{array}$} & $\begin{array}{c}\mathrm{F} \\
n=102\end{array}$ & $\begin{array}{c}14 \\
(13,73)\end{array}$ & $\begin{array}{c}84 \\
(82,35)\end{array}$ & $\begin{array}{c}3 \\
(2,94)\end{array}$ & $\begin{array}{c}1 \\
(0,98)\end{array}$ \\
\hline & $\begin{array}{c}M+F \\
n=318\end{array}$ & $\begin{array}{c}46 \\
(14,47)\end{array}$ & $\begin{array}{c}262 \\
(82,39)\end{array}$ & $\begin{array}{c}8 \\
(2,51)\end{array}$ & $\begin{array}{c}2 \\
(0,63)\end{array}$ \\
\hline & $\begin{array}{c}\mathrm{M} \\
n=186\end{array}$ & $\begin{array}{c}51 \\
(27,42)\end{array}$ & $\begin{array}{c}133 \\
(71,50)\end{array}$ & $\begin{array}{c}2 \\
(1,08)\end{array}$ & \\
\hline \multirow[t]{3}{*}{$\begin{array}{l}\text { Saison sèche chaude } \\
\text { (fév. mars. avril) }\end{array}$} & $\begin{array}{c}\mathrm{F} \\
n=107\end{array}$ & $\begin{array}{c}27 \\
(25,23)\end{array}$ & $\begin{array}{c}78 \\
(72,90)\end{array}$ & $\begin{array}{c}2 \\
(1,87)\end{array}$ & \\
\hline & $\begin{array}{c}\mathrm{M}+\mathrm{F} \\
n=293\end{array}$ & $\begin{array}{c}78 \\
(26,62)\end{array}$ & $\begin{array}{c}211 \\
(72,01)\end{array}$ & $\begin{array}{c}4 \\
(1,37)\end{array}$ & \\
\hline & $\begin{array}{c}\mathrm{M} \\
n=568\end{array}$ & $\begin{array}{c}70 \\
(12,32)\end{array}$ & $\begin{array}{c}445 \\
(78,35)\end{array}$ & $\begin{array}{c}48 \\
(8,45)\end{array}$ & $\begin{array}{c}5 \\
(0,88)\end{array}$ \\
\hline \multirow[t]{2}{*}{$\begin{array}{l}\text { Saison pluvieuse } \\
\text { (mai à octobre) }\end{array}$} & $\begin{array}{c}\mathrm{F} \\
n=201\end{array}$ & $\begin{array}{c}30 \\
(14,93)\end{array}$ & $\begin{array}{c}155 \\
(77,11)\end{array}$ & $\begin{array}{c}13 \\
(6,47)\end{array}$ & $\begin{array}{c}3 \\
(1,49)\end{array}$ \\
\hline & $\begin{array}{c}\mathrm{M}+\mathrm{F} \\
n=769\end{array}$ & $\begin{array}{c}100 \\
(13,00)\end{array}$ & $\begin{array}{c}600 \\
(78,02)\end{array}$ & $\begin{array}{c}61 \\
(7,94)\end{array}$ & $\begin{array}{c}8 \\
(1,04)\end{array}$ \\
\hline
\end{tabular}

0,1-1 cm: diamètre du support ligneux (branches, brindilles, tronc) de repos, compris entre 0,1 et $1 \mathrm{~cm}$ inclus. $\mathrm{M}=$ mâle; $\mathrm{F}=$ femelle; $\mathrm{M}+\mathrm{F}=$ mâle et femelle, $n=$ nombre total de glossines mâles, femelles ou males et femelles, observées au repos sur support ligneux durant la saison considérée. Les chiffres entre parenthèses représentent les pourcentages de glossines observées au repos, ces pourcentages étant, dans chaque catégorie $(\mathrm{M}, \mathrm{F}, \mathrm{M}+\mathrm{F})$, calculés par rapport aux valeurs correspondantes de $n$. 
Comme l'indique le Tableau 3, des glossines ont été trouvées au repos jusqu'à $1,50 \mathrm{~m}$ de hauteur quelle que soit la saison et quel que soit leur sexe; entre 1,5 et $2 \mathrm{~m}$ de hauteur, les mouches n'ont été observées au repos qu'en saison sèche froide et en saison des pluies. C'est seulement en saison pluvieuse que les mouches tsétsé ont été repérées au repos entre 2 et $2,50 \mathrm{~m}$ avec ne proportion de mâles nettement plus élevée ( $2 \%$ ) que celle des femelles (moins de $1 \%$ ).

Quelle que soit la saison (voir Tableau 3), plus de la moitié des mouches se reposaient à une hauteur inférieure à $50 \mathrm{~cm}$. On a noté cependant en saison sèche chaude, une proportion de mouches au repos entre 1 et $50 \mathrm{~cm}$ du sol nettement plus importante (73\%) que celles notées aux mêmes hauteurs en saison sèche froide $(57 \%)$ et en saison pluvieuse $(51 \%)$.

Répartition des glossines au repos en fonction du diamètre du support ligneux: variations saisonnières

Comme l'indique le Tableau 4, les diamètres des supports ligneux de repos de $G$. palpalis palpalis dans notre zone d'étude allaient, dans l'ensemble de $0,1 \mathrm{~cm}$ (brindilles d'Eupatorium odoratum ou d'Acacia pennata par exemple) à $8 \mathrm{~cm}$ (branches de caféiers ou de cacaoyers par exemple).

Quelleque soit la saison, plus de $90 \%$ des mouches (97\% en saison sèche froide; $99 \%$ en saison sèche chaude ; $91 \%$ en saison pluvieuse) se reposaient sur des supports dont les diamètres étaient inférieurs ou égaux à $3 \mathrm{~cm}$ avec les plus fortes proportions (plus de $75 \%$ en moyenne en toutes saisons) enregistrées sur les supports de diamètres allant de 1,1 à $3 \mathrm{~cm}$.

On note cependant (voir Tableau 4), que la proportion de mouches au repos sur les supports dont les diametres allaient de $0,1 \mathrm{a} 1 \mathrm{~cm}$ (supports de très faibles diametres) etait nettement plus élevée en saison sèche chaude $(27 \%)$ qu'en saison sèche froide (14\%) ou en saison pluvieuse (13\%).

On note enfin, toujours à l'analyse du Tableau 4, que les plus gros supports ligneux (diamètres allant de 5,1 à $8 \mathrm{~cm}$ ) n'ont hébergé que très peu de glossines: moins de $1 \%$ en moyenne en saison sèche froide et en saison pluvieuse alors qu'en saison sèche chaude, aucune mouche n'a été observée au repos sur ces gros supports.

Collecte de repas sanguins frais pour identification des hôtes naturels de G. palpalis palpalis

Sur les 938 glossines effectivement capturées au repos, 250 soit environ $27 \%$, ont été trouvées gorgées ou en phase intermédiaire et ont pu ainsi procurer, après dissection, des repas de sang frais pour identification ultérieure des hôtes naturels et la mise en évidence des préférence trophiques de G.palpalis palpalis dans la zone d'étude.

\section{DISCUSSIONS: INTERET ET APPLICATION DE CETTE ETUDE}

\section{Pourquoi une telle étude?}

Depuis la découverte d'insecticides de synthèse à effet rémanent et leur pulvérisation surtout à partir de 1945 dans les habitats naturels des glossines, les lieux de repos diurnes et nocturnes de ces diptères hématophages, firent l'objet d'innombrables travaux de recherche dont les revues ont été publiées par Langridge et al. (1963), Jordan (1974), Hadaway (1977), Challier (1982).

D'une facon générale, il ressort de ces travaux que: (1) les glossines, dans le choix de leur substrat de repos, ne semblent montrer aucune préférence particulière pour telle ou telle espèce de plantes. Cependant, dans des conditions locales particulières, elles sont presque toujours observées ou capturées au repos sur les mêmes espèces de plantes (Laveissière, 1976 b; Lavaissière et al., 1979 b; Gruvel, 1975; Turner, 1980 c; Scholtz et al., 1976).

(2) durant la journée, les mouches se reposent de préférence sur des supports ligneux généralement inclinés (branches, brindilles, troncs) avec une prédilection pour la face inférieure de ces substrats (Abdurrahim, 1971; Okiwelu, 1981, 1983).

(3) les lieux de repos nocturnes sont constitués en majorité de feuilles, la glossine marquant une préférence pour la face supérieure de ces feuiles (Challier, 1973).

(4) la hauteur de repos des glossines est en général plus faible de jour que de nuit; de même, les lieux de repos sont, dans l'ensemble plus proches du sol en saison sèche qu'en saison pluvieuse.

Malgré ces quelques généralisations qui peuvent être faites à partir des recherches antérieures, tout épandage rationnel d'insecticide à effet remanent dans les habitats des glossines dans un foyer de maladie du sommeil donné, nécessite malheureusement encore une étude sur les lieux de repos diurnes et/ou nocturnes des vecteurs concernés. Ceci, pour tenir compte des changements possibles de comportement de ces vecteurs d'une espèce de glossine à une autre, d'une région à une autre ou d'un biotope à un autre au sein d'une même region bioclimatique.

En guise d'exemples illustrant cette possibilité pour la glossine de changer son comportement de repos, évoquons, en les comparant à ceux de nos 
présentes études, quelques résultats obtenus par d'autres auteurs sur $G$. tachinoides, $G$. palpalis gambiensis et $G$. palpalis palpalis, les trois vecteurs connus de la trypanosomiase humane a $T . b$. gambiense en Afrique Occidentale.

Des études ont été menées sur les lieux de repos diurnes de $G$. tachinoides en savane guinéenne durant la saison sèche chaude (Laveissière et al., 1978). Ces travaux ont conduit leurs auteurs à faire les recommandations suivantes en matière de pulvérisation d'insecticides rémanents contre $G$. tachinoides dans leur zone d'étude :

(1) traiter la végétation sur une hauteur comprise entre 0 et $80 \mathrm{~cm}$ du sol;

(2) traiter "les organes ligneux d'un diamètre supérieur à $10 \mathrm{~cm}$ en insistant sur les trous d'arbres".

Or, comme nous l'avons mentionné plus haut, $G$. palpalis palpalis dans le foyer de Bouaflé a, quant à elle, été trouvée au repos en saison sèche chaude jusqu'à 1,50 m de hauteur, sur des supports ligneux dont les diamètres ne dépassaient pas $5 \mathrm{~cm}$ et n'a jamais été trouvée se reposant dans des creux d'arbres.

Nous voyons donc que les recommandations formulées par ces auteurs pour lutter contre $G$. tachinoides en savane soudanienne, ne sauraient être appliquées pour lutter efficacement contre G. palpalis palpalis dans la zone préforestière où se sont déroulées nos études.

S'agissant de G. palpalis gambiensis, aucun specimen de cette espèce n'a été trouvée de jour au repos à plus de $1 \mathrm{~m}$ du sol durant la saison sèche froide (décembre) dans une galerie forestière de savane guinéenne (Bois et al., 1977). Par contre, dans notre zone d'étude (voir Tableau 3), 84\% environ des spécimens de $G$. palpalis palpalis se reposaient en saison sèche froide entre 0 et $1 \mathrm{~m}$ du sol et $16 \%$ environ furent repérées au repos à plus de $1 \mathrm{~m}$ du sol.

Des travaux sur les lieux de repos diurnes de $G$. palpalis palpalis ont été conduits dans le foyer de trypanosomiase humaine de Vavoua, en secteur forestier de Côte d'Ivoire(Gouteux, 1984). Ces études ont montré qu'en saison sèche, sur 56 glossines trouvées au repos sur supports ligneux, 38 , soit environ $68 \%$, avaient choisi des supports de plus de $10 \mathrm{~cm}$ de diamètre alors que nos présentes études ont révélé qu'en secteur préforestier (foyer de Bouaflé), 97 à $99 \%$ des glossines de cette même espèce se reposaient de jour et en saison sèche sur des supports ligneux de diamètres inférieurs à $3 \mathrm{~cm}$ et que par ailleurs les supports de diamètres supérieurs à $8 \mathrm{~cm}$ n'ont hébergé aucun specimen de cette espèce.

On perçoit donc aisément à travers ces trois exemples, le bien-fondé de notre étude dont l'intérêt réside non seulement dans la confirmation dans notre zone d'étude des quelques principes généraux déjà acquis sur les lieux de repos et le comportement de la glossine au repos, mais aussi et surtout dans la mise en évidence des spécificités caractérisant le vecteur au repos dans l'environnement bio-écologique considéré. Ce sont ces spécificités, beaucoup plus que les généralités, qui vont nous guider dans l'adoption des tactiques de lutte chimique à appliquer dans le foyer contre le vecteur incriminé.

\section{Sur le sex-ratio de la population de glossines au repos}

Le sex-ratio, défini ici comme le pourcentage de femelles, est d'environ $30 \%$ par rapport à l'ensemble des glossines observées au repos.

Dans tous les biotopes explorés et quelle que soit la saison, le nombre de mâles observés au repos a toujours été nettement plus élevé (supérieur à $60 \%$ ) que celui des femelles alors que les captures effectuées dans la zone d'étude à l'aide des pièges biconiques Challier-Lavaissière (Challier et Lavaissière, 1973) à la lisière des villages à forte densité de porcs par exemple, révèlent tout au long de l'année des sexratio supérieurs à $50 \%$ avec des valeurs pouvant excéder $75 \%$ en certaines saisons (Sékétéli, communication personnelle).

Kupper (1983), Kupper et Koch (1983), ont noté lors d'étude sur les lieux de repos nocturnes de $G$. palpalis gambiensis et $G$. tachinoides dans l'ouest du Burkina Faso (ex-Haute Volta), que les mâles retrouvés au repos étaient plus nombreux que les femelles. Les études sur les lieux de repos diurnes de G. tachinoides en savane guinéenne (Laveissière et al., 1978), font état d'un sex-ratio de la population de mouches capturées au repos en saison sèche chaude d'environ $47 \%$.

Ce faible taux de femelles observées au repos dans notre zone d'étude pourrait s'expliquer par le fait que les heures que nous avons choisies pour ces recherches (entre 11h et 15h 30) ne correspondent pas forcément aux heures préférentielles de repos diurnes des femelles: en effet, ces heures semblent représenter également la période de plus intense activité des mouches si on en juge par les captures élevées notées dans les pièges biconiques dans la zone d'étude durant cette partie de la journée (Sékétéli, communication personnelle). On pourrait donc émettre l'hypothèse que durant cette période chaude de la journée, une fraction importante de femelles est plus préoccupée que les mâles à voler à la recherche de ce repas sanguin indispensable à la maturation de ses oeufs. De plus, les femelles gravides pourraient, durant cette même période de la journée, se trouver ailleurs qu'à leurs lieux de repos, soit pour déposer leurs larves ou pour rechercher un lieu propice à cette larviposition. 
Lieux de repos diurnes, gîtes à pupes et terrains de chasse des glossines

Dans notre zone d'étude, les pupes ont été récoltées dans les plantations de café, de cacao, dans les champs en jachère et sous la végétation des lisières des villages constituée essentiellement d'Acacia pennata, d'autres lianes et d'Eupatorium odoratum (Sékétéli et Kuzoe, 1984).

Ce sont ces mêmes types de végétation qui ont constitué les lieux de repos diurnes de G. palpalis palpalis dans cette zone. Ce phénomène semble plus marqué dans les localités à forte densité de porcs domestiques, où il arrive souvent que les glossines se reposent directement sur les mêmes plantes sous lesquelles les femelles vont déposer leurs larves. Par ailleurs, les abris constitués par ces plantes sont souvent fréquentés par les porcs domestiques, hôtes préférés de $G$. palpalis palpalis dans la zone (Fig. 3). Cette disponibilité de l'hôte préféré va constituer un facteur qui pourrait influencer fortement le choix du lieu de repos diurne de la glossine (Laveissière et al. 1981).

Ces localités où abondent les porcs vont par conséquent représenter pour les glossines, à la fois des terrains de chasse privilégiés pour la recherche de la nourriture, des lieux de ponte et de repos, réunissant ainsi pour $G$. palpalis palpalis toutes les conditions favorables pour un comportement péridomestique (Baldry, 1968a, b et 1980; Eouzan et Ferrara, 1978; Sékétéli et Kuzoe, 1984).

Capture des glossines au repos: un excellent moyen de collecte de repas sanguin pour l' identification des hôtes naturels de G. palpalis palpalis

La recherche à vue, sans marquage, des glossines au repos est une entreprise certes pénible qui demande du temps et surtout de la patience de la part des observateurs. Cependant, dès que les lieux sont répérés après parfois plusieurs jours de tâtonnement, l'opération peut s'avérer rentable si on en juge par les résultats que nous avons obtenus: 183 mouches observées au repos pour 100 heures de recherche ce qui représente 5 jours de recherche par une équipe de 5 personnes travaillant $4 \mathrm{~h}$ en moyenne par jour!

Par ailleurs, la technique de capture des mouches au repos à l'aide des tubes de verre a été efficace puisqu'elle nous a permis de capturer en toutes saisons plus de $60 \%$ des glossines trouvées au repos.

Si nous tenons compte de fait qu'environ $27 \%$ des mouches capturées au repos ont procuré dans la zone d'étude des repas de sang frais pour identification alors que là $2 \%$ seulement de ces glossines capturées à l'aide des pièges biconiques Challier-Laveissière furent en état de procurer des repas de sang frais identitifiables par les techniques actuelles (Sékétéli, communication personnelle), nous pouvons dire que la capture des glossines au repos dans leurs habitats constitue, du moins dans notre zone d'étude, un moyen privilégié pour la collecte de repas de sang frais en vue de l'identification des hôtes naturels de G. palpalis palpalis.

Données de littérature sur les lieux de reposnocturnes de G. palpalis palpalis dans le foyer de Bouaflé

La première et la seule étude effectuée avant la nôtre sur les lieux de repos de G. palpalis palpalis dans le foyer de Bouaflé fut celle de 1976 menée dans une portion de galerie forestière le long du fleuve Marahoué, principal cours d'eau drainant la zone d'étude (Laveissière et Challier 1976). Cette étude de 1976 avait trait uniquement au lieu de repos nocturne du vecteur à partir de 1.158 glossines marquées dont seulement 42 individus ont pu être recapturés. Elle a abouti aux résultats suivants: 69\% des glossines retrouvées se reposaient sur des feuilles et des herbes, $26 \%$ d'entre-elles ont choisi des supports ligneux (petites branches, brindilles) pour leur repos, $5 \%$ des glossines furent retrouvées au sol et les auteurs pensent qu'il s'agissait d'individus en mauvais état n'ayant pas pu voler; quant à la hauteur de repos, toutes les glossines ont été retrouvées à moins de $1 \mathrm{~m}$ du sol avec $93 \%$ d'entre-elles se reposant à moins de $50 \mathrm{~cm}$ du sol et enfin, $83 \%$ d'entre-elles furent repérées entre 0 et $7 \mathrm{~m}$ de l'eau.

Nous tiendrons également compte de ces données dans les recommandations que nous allons formuler pour l'application d'insecticides rémanents dans l'habitat de G.palpalis palpalis dans la zone d'étude.

Recommandations pour l'épandage d'insecticides rémanents contre les vecteurs de la maladie $d u$ sommeil dans le foyer de Bouaflé

L'efficacité d'un traitement insecticide rémanent va dépendre de la possibilité, pour la glossine, de se trouver en contact d'une surface traitée sur laquelle elle pourra prélever une dose léthale (Challier et al., 1978). La connaissance des lieux de repos dans notre zone d'étude va donc nous guider à appliquer les insecticides remanents aux endroits qui sont les plus susceptibles d'accroître la probabilité de ce contact du vecteur avec le dépôt insecticide.

Pour l'application d'insecticides rémanents contre les vecteurs de la trypanosomiase humaine dans le foyer de Bouaflé, nous faisons donc les recommandations suivantes:

(a) Ce qui' il ne faut pas faire. (1) Ne pas traiter en saison pluvieuse. (2) Ne pas traiter les gros troncs d'arbre de diamètres supérieurs à $8 \mathrm{~cm}$. 
(3) Ne pas traiter l'axe principal et vertical d'un caféier ou d'un cacaoyer. (4) Ne pas épandre l'insecticide sur les feuilles, les herbes, les petites branches ou les brindilles qui jonchent le sol. (5) Ne pas traiter les arbres isolés au milieu des villages et qui servent à procurer de l'ombre aux habitants.

(b) Ce qui' il faut faire. (1) Traiter en saison sèche et de préférence en saison sèche froide (novembre, décembre ou janvier), (2) Traiter toute la végétation bordant les villages, les sentiers, les grands axes routiers en mettant l'accent sur les lianes telles que Acacia pennata, sur les Composées telles que Eupatorium odoratum. (3) Dans les plantations de café et de cacao ne traiter que les ramifications en mettant l'accent sur les branches inclinées. (4) Dans tous lescas, bien traiter la face inférieure des organes ligneux de faibles diamètres (brindilles, branche, tiges inclinées de 0,1 à 3 $\mathrm{cm}$ de diamètre). (5) Traiter la plante visée jusqu'à une hauteur de $3 \mathrm{~m}$ au dessus de sol. (6) Faire pénétrer l'insecticideà travers la végétation sur une largeur de 5 à $7 \mathrm{~m}$.

Toutes ces recommandations ont pu être déjà appliquées avec succès lors des épandages au sol de pyréthrinoides de synthèse que nous avons effectuées dans la zone d'étude non seulement contre $G$. palpalis palpalis mais également contre $G$. tachinoides, deuxième vecteur de la maladie du sommeil dans le foyer considéré (Sékétéli et Kuzoe, 1986).

Remerciements-Les résultats des présents travaux représentent le fruit de la patience et de la dextérité de messieurs Coulibaly Siaka, Sanon Borema, Dabiré Jacques, Nankodaba Gnihan, Kouakou oi Kouakou, Konan Kouadio, Fofana Korofo et Kouadiani Jules. Qu'ils trouvent tous ici l'expression de notre profonde gratitude.

Nos sincères remerciements vont également au Dr. P. de Raadt qui n'a cessé d'encourager ces genres d'études et au Dr. René Le Berre pour ses conseils et sa lecture critique du manuscrit.

\section{REFERENCES}

Abdurrahim V. (1971) A study of the diurnal resting behaviour of Glossina palpalis in Southern Zaria, Northern Nigeria. ISCTRC, 13th Meeting. OAU/ STRC. 105, pp. 213-227.

Baldry D. A. T. (1968a) The epidemiological significance of recent observations in Nigeria on the ecology of Glossina tachinoides Westwood (Diptera: Muscidae). Bull. entomol. soc. Nigeria 2, 34-38.

Baldry D. A. T. (1968b) Observations on the peridomestic breeding behaviour and resting sites of Glossina tachinoides Westw. near Nsukka, East Central State, Nigeria. Bull. entomol. Res. 59, 585-593.

Baldry D. A. T. (1980) Local distribution and ecology of Glossina palpalis and $G$. tachinoides in forest foci of West African human trypanosomiasis, with special reference to associations between peridomestic tsetse and their hosts. Insect Sci. Applic. 1, 85-93.

Bois J. F., Challier A., Laveissière C. et Ouédraogo V. (1977) Recherche des lieux de repos diurnes des glossines (Glossina palpalis gambiensis Vanderplank, 1949: Diptera, Glossinidae) par détection de specimens marqués au $59 \mathrm{Fe}$. Cah. ORSTOM sér. entomol. med.Parasitol. 15,3-13.

Challier A. (1973) Ecologie de Glossina palpalis gambiensis Vanderplank, 1949 (DipteraMuscidae) en savane d'Afrique Occidentale. Memoires ORSTOM 64.

Challier A. (1982) The ecology of tsetse (Glossina spp.) (Diptera, Glossinidae) : a review (19701981). Insect Sci. Applic. 3, 97-143.

Challier A. et Laveissière C. (1973) Un nouveau piège pour la capture des glossines (Glossina: Diptera, Muscidae): description et essais sur le terrain. Cah. ORSTOM Sér. entomol. méd. Parasitol. 11, 251-262.

Challier A., Eyraud M. et Laveissière C. (1978) Application sélective d'insecticides rémanents aux lieux de repos des glossines riveraines: essais en zone de savane guinéenne et possibilités d'application dans les différentes zones bioclimatiques d'Afrique Occidentale. Cah. ORSTOM Sér. entomol. med. et Parasitol. 16,515.

Eouzan J. P. et Ferrara L. (1978) Comportement péridomestique de Glossina palpalis palpalis (R.D.) dans un foyer de maladie du sommeil au Cameroun. Cah. ORSTOM, sér. Ent. Méd. Parasitol. 16, 237-242.

Gouteux J. P. (1984) Ecologie des glossines en secteur préforestier de Côte d'Ivoire. Relation avec la trypanosomiase humaine et possibilités de lutte. Thèse doctorat d'Etat. Université ParisSud, Centre d'orsay No. d'ordre 2835.

Gruvel J. (1975) Lieux de repos de G. tachinoides W. Rev. Elev. Méd. Vét. Pays trop. 28, 158-172.

Hadaway A. B. (1977) Resting behaviour of tsetse flies and its relevance to their control with residual insecticides. Miscellaneous Report No. 36. COPR, London.

Jordan A. M. (1974) Recent developments in the ecology and methods of control of tsetse flies (Glossina spp.) (Diptera: Glossinidae), a review. Bull. entomol. Res. 63, 361-399.

Kupper W. et Koch K. (1983) Les gîtes de repos 
nocturnes de Glossina spp. (Diptera: Glossinidae) dans l'Ouest de la Haute-Volta. I. Glossina tachinoides, Westwood. Rev. Elev. Méd. Vét. Pays trop. 36, 175-181.

Kupper W. (1983) Les gîtes de repos nocturnes de Glossina spp. (Diptera: Glossinidae) dans l'Ouest de Ia Haute-Volta. II. Glossina palpalis gambiensis Vanderplank. Rev. Elev. Méd. Vét. Pays trop. 36, 265-271.

Langridge W. P., Kernaghan R. J. et Glover P. E. (1963) A review of recent knowledge of the ecology of the main vectors of trypanosomiasis. Bull. WHO 28, 671-701.

Laveissière C. (1976b) Ecologie de Glossina tachinoides Westwood en savane humide d'Afrique de l'Ouest. II. Lieux de repos nocturnes. Cah. ORSTOM, sér. Entomol. Med. Parasitol. 14, 331-345.

Laveissière C. et Challier A. (1976) Le foyer trypanosomiase humaine de Bouaflé (Côte d'Ivoire): enquête entomologique et épidémiologique, propositions pour une campagne insecticide. Doc. tech. OCCGE 6308. multigr

Laveissière C., Kiénou J. P. et Traore T. (1979b) Ecologie de Glossina tachinoides Westwood, 1850 , en savane humide d'Afrique de l'Ouest. VII. Lieux de repos diurnes, variations saisonnières. Cah.ORSTOM,Sér.Entomol.Med. Parasitol. 17, 181-191.

Laveissière C. , Kiénou J. P., Ouedraogo K. V. et Traore T. (1978) Lieux de repos diurnes de Glossina tachinoides Westwood (savane guinéenne, saison chaude). Cah. ORSTOM, Ser. Entomol. Med. Parasitol. 16, 3-71.

Laveissière C., Kiénou J. P. et Traore T. (1981) Ecologie de Glossina tachinoides Westwood, 1850, en savane humide d'Afrique de l'Ouest. VIII. Facteurs influençant le choix d'un lieu de repos diurne. Cah. ORSTOM Sér. Entomol.Med. Parasitol. 19, 261-269.

Okiwelu S. N. (1981) Resting site preferences of the tsetse Glossina palpalis gambiensis Vanderplank (Diptera:Glossinidae), in Mali.Insect Sci.Applic.
1, 289-294.

Okiwelu S. N. (1983) Further observations on the resting site preferences of the tsetse, Glossina palpalis gambiensis Vanderplank (Diptera: Glossinidae), in Mali. Insect Sci. Applic. 4, 305308.

Scholtz E., Spielberger U. and Ali J. (1976) The night resting sites of the tsetse fly Glossina palpalis palpalis (Robineau-Desvoidy) (Diptera: Glossinidae) in northern Nigeria. Bull. Entomol. Res. 66, 443-452.

Sékétéli A.et KuzoeF. A. S. (1983)Essais d'épandage au sol de pyréthrinoïdes de synthèse (cyperméthrine, décaméthrine, perméthrine) contre Glossina palpalis s.l. dans une zone préforestière de Côte d'Ivoire: Caractéristique physique des gouttelettes et effet sur la densité des populations vectrices. ISCTRC 17th Meeting. OAU/STRC 112, 589-608.

Sékétéli A. et Kuzoe F. A. S. (1983) Gîtes à pupes de Glossina palpalis s.l. dans une zone préforestière de Côte d'Ivoire. Acta Tropica 41, 293-301.

Sékétéli A., Johannes L., Van de Laar M. et Kuzoe F. A. S. (1985) Essais d'épandage au sol de la deltaméthrine poudre mouillable à différentes doses contre Glossina palpalis s.l. dans une zone préforestière de Côte d'Ivoire. Insect Sci. Applic. 6, 187-192.

Sékétéli A. et Kuzoe F. A. S. (1986) Effet résiduel sur Glossina palpalis palpalis de 1'alphamethrine (pyrétrinoïde de synthèse) en concentré émulsifiable $10 \%$ appliqué par épandage au sol en zone préforestière de Côte d'Ivoire. Insect Sci. Applic. 7, 757-761.

Sékétéli A. et KuzoeF.A.S.(1986)Essais d'épandage au sol de trois pyrethrinoides de synthèse (OMS 2012 p.m. 10\%, OMS 2013 p.m. 5\%, OMS 3004 p.m. 5\%) contre Glossina palpalis palpalis et Glossina tachinoides en zone préforestière de Côte d'Ivoire. Insect Sci. Applic. 7, 763-769.

Turner D. A. (1980c) Tsetse ecological studies in Niger and Mozambique. II. Resting behaviour. Insect Sci. Applic. 1, 15-21. 